\title{
Du printemps ouvrier à l'altermondialisme... Le champ militant et le champ politique en Turquie
}

From a working class spring to alter-globalisation. The militant field and the political field in Turkey

Gülçin Erdi Lelandais

\section{OpenEdition}

Journals

Édition électronique

URL : http://journals.openedition.org/conflits/12483

DOI : $10.4000 /$ conflits. 12483

ISSN : $1777-5345$

Éditeur :

CCLS - Centre d'études sur les conflits lilberté et sécurité, L'Harmattan

Édition imprimée

Date de publication : 20 juillet 2008

Pagination : $57-83$

ISBN : 978-2-296-05909-2

ISSN : $1157-996 \mathrm{X}$

\section{Référence électronique}

Gülçin Erdi Lelandais, « Du printemps ouvrier à l'altermondialisme... Le champ militant et le champ politique en Turquie », Cultures \& Conflits [En ligne], 70 | été 2008, mis en ligne le 10 septembre 2008, consulté le 30 mars 2021. URL : http://journals.openedition.org/conflits/12483 ; DOI : https://doi.org/ $10.4000 /$ conflits. 12483 


\title{
Du printemps ouvrier à l'altermondialisme... Le champ militant et le champ politique en Turquie
}

\section{Gülçin ERDI LELANDAIS}

Gülçin Erdi Lelandais est docteure en sociologie (EHESS), chercheuse associée an Centre d'études sur les conflits et au Laboratoire de sciences sociales du politique (LaSSP). Ses travaux portent sur l'altermondialisme, la transnationalisation des monvements sociaux, la sociologie du risque et les mouvements sociaux et politiques en Turquie.

\begin{abstract}
« Notre rôle, c'est de casser dans la tête des individus l'idée que tout est comme ça et que rien ne pourrait changer. Il faut arriver à convaincre les gens que si on se réunit, si on résiste, tout est possible. C'était le cas du $1^{\text {er }}$ mars [1]. Tout le monde a cru que le Parlement voterait naturellement pour la participation à la guerre en Irak, mais toutes les organisations de la société civile, ensemble, ont organisé une mobilisation tellement forte que ça a cassé l'image dans l'esprit des gens que la mobilisation sociale ne peut jamais affecter les politiques gouvernementales ${ }^{2}$.»
\end{abstract}

\footnotetext{
Ces paroles d'un militant altermondialiste rencontré en Turquie nous pro-

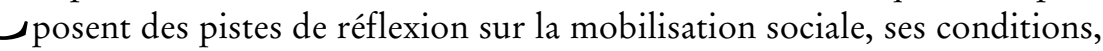
sa réalisation et les interactions entre le champ politique et le champ militant dans ce pays. Elles attirent également notre intention sur une reconfiguration certes lente, mais bien réelle, du système socio-politique turc concernant la position de divers acteurs à la fois politiques, sociaux et économiques.
}

En raison de sa candidature à l'Union européenne et des négociations ouvertes avec cette dernière, la Turquie poursuit des réformes dans tous les

1. er $^{\mathrm{er}}$ mars 2003, date du vote au Parlement turc pour l'envoi de troupes militaires turques en Irak, aux côtés des Etats-Unis.

2 . Propos recueillis lors de notre entretien avec le porte-parole de l'Antikapitalist, Sertug Cicek, le 9 décembre 2003, Ankara. 
domaines. Depuis 2002, le nombre de modifications de lois et d'amendements est considérable et le pays s'apprête à adopter une nouvelle Constitution dans les mois à venir. Dans ce contexte, les anciennes configurations du champ politique sont bousculées, ce qui n'est pas sans créer des crises politiques fréquentes ${ }^{3}$ en raison de la lutte entre les acteurs, soit pour préserver leur place existante, soit pour en obtenir une dans le champ politique du pays.

L'émergence de l'altermondialisme en tant que nouvelle cause de lutte montre la transformation du champ des luttes en lien avec les évolutions politiques et économiques, tant en Turquie qu'au niveau international, notamment par l'accentuation de la mondialisation.

Nous proposons dans cet article de conduire une réflexion sur les conditions de mobilisation de l'altermondialisme dans ce climat de reconfiguration du champ politique en Turquie, en examinant d'un côté les conditions politiques et, de l'autre, les configurations du champ militant turc qui peuvent constituer à la fois une opportunité et un obstacle à la mobilisation.

\section{Configuration du champ politique en Turquie depuis 1980}

Le champ politique, d'après Pierre Bourdieu, peut être défini «à la fois comme champ de forces et comme champ des luttes visant à transformer le rapport de forces qui confère à ce champ sa structure à un moment donné 4 ». Un champ politique est donc un lieu de lutte entre les différents agents occupant diverses positions dont l'enjeu est l'appropriation ou la redéfinition d'un capital spécifique au champ. Chaque champ possède des règles du jeu et des enjeux spécifiques, irréductibles aux règles du jeu et enjeux des autres champs 5 . Selon Bernard Lahire, "les acteurs de ce champ partagent une complicité objective de jouer le jeu - illusio-par-delà les luttes qui les opposent ${ }^{6}{ }^{»}$.

A la lumière de ces caractéristiques, nous pouvons tenter de définir le champ politique turc. L'effondrement de l'Empire ottoman et la proclamation de la République laïque par Mustafa Kemal constituent le moment fondateur

3. Les crises politiques opposent particulièrement les laïcs-kémalistes aux islamistes, à savoir le gouvernement en place. Une crise politique a récemment eu lieu suite à l'élection du président de la République. L'armée et les kémalistes étaient opposés à l'élection du candidat de l'AKP (Parti de la justice et du développement), islamo-conservateur au pouvoir, dont la femme porte le voile islamique. Récemment, le procureur du Conseil suprême a déposé une plainte pour la fermeture de l'AKP, le considérant comme le «centre » des activités islamistes. Pour de plus amples informations sur ce sujet, voir Erdi Lelandais G., «L'énigme de l'AKP : regards sur la crise politique en Turquie », Politique étrangère, n`3, 2007, pp. 547-560.

4. Bourdieu P., "La représentation politique. Eléments pour une théorie du champ politique », Actes de le recherche en sciences sociales, n³6 -37, février-mars 1981, pp. 3-24.

5. Lahire B., "Champ, hors-champ, contrechamp ", Le Travail sociologique de Pierre Bourdieu. Dettes et critiques, Paris, La Découverte, 2001, p. 24.

6. Ibid., p. 25. 
de celui-ci. Le contexte de la guerre de libération (1919-1923) menée contre les pays européens à la fin de la Première Guerre mondiale a conféré à ce champ les mots d'ordre suivants : le patriotisme et le nationalisme. La laïcité vient s'ajouter à ces deux principes à partir de 1928, avec l'abolition du califat. Ces trois principes fondamentaux représentent encore aujourd'hui les conditions sine qua non d'accès au champ politique turc. L'autonomisation du champ politique s'accélère après la mise en place du multipartisme en 1946. Depuis lors, le champ politique turc témoigne d'une véritable lutte symbolique entre les acteurs non seulement établis dans le champ mais également entre ces derniers et les acteurs tentant d'y accéder. A différentes périodes de l'histoire politique de la Turquie, les courants politiques socialistes et communistes, puis islamistes conservateurs ont tenté d'obtenir une place dans ce champ. Les partis politiques nationalistes et kémalistes y seront les acteurs permanents.

Néanmoins, nous sommes loin de pouvoir constater une stabilisation du champ politique en Turquie. Il nous faut d'ailleurs en souligner quelques spécificités. Tout d'abord, à l'intérieur du champ politique turc, et contrairement à la thèse de Bourdieu 7, les acteurs ne sont pas toujours des professionnels, le système des grandes écoles administratives de type ENA n'existant pas. Très souvent, députés et ministres n'ont pas de diplôme d'enseignement supérieur. Adhérant à l'illusio du champ - le nationalisme -, certains acteurs et organisations sont susceptibles d'avoir un succès dans ce champ ${ }^{8}$. Autrement dit, la différence entre professionnels et "profanes» n'est pas entièrement tranchée. L'origine socioprofessionnelle des acteurs politiques est très diverse ${ }^{9}$. Le clivage entre les élites « rurales » et les élites « urbaines » appartenant à des classes moyennes supérieures des grandes villes est particulièrement important dans la configuration des partis politiques ${ }^{10}$. Le champ politique n'a, par ailleurs, pas encore réalisé son autonomisation complète, notamment vis-à-vis du champ militaire. L'armée constitue un acteur à part entière de ce champ et la continuité des autres acteurs en son sein dépend de leur positionnement à l'égard de cette dernière. Cette place prépondérante de l'armée a engendré à trois reprises ${ }^{11}$ des

7. Bourdieu P., Propos sur le champ politique, Lyon, Presses universitaires de Lyon, 2000, p. 55.

8. Ce constat a été illustré par l'exemple du Genç Parti («Parti jeune ») lors des élections législatives de 2002. Son président, ex-propriétaire d'une entreprise de téléphonie mobile, milliardaire, condamné pour fraude fiscale et détournement d'argent, a réussi à obtenir $7 \%$ des voix en se basant uniquement sur un discours nationaliste et en utilisant le drapeau turc (croissantétoile) comme logo de son parti.

9. Il y a très peu de recherches sur l'origine socio-professionnelle des élus en Turquie. De rares études nous fournissent des informations datant des années 1970, qui ne sont plus valables aujourd'hui. Cependant, la liste des élus, en automne 2007, nous fournit quelques indices sur ce sujet. $10 \%$ des élus sont diplômés de l'enseignement primaire et $60 \%$, de l'enseignement supérieur. Le nouveau Parlement, après les élections législatives de juillet 2007, ne représente que $6 \%$ des élus diplômés du primaire et du secondaire (voir : http://www.memurlar.net/haber/83265/, consulté le 11/04/2008).

10. Ünsal A., Siyaset ve Anayasa Mabkemesi (Politique et Cour constitutionelle), Ankara, SBF Yayinlari, 1980, p. 220.

11. L'armée turque a eu recours en 1960, en 1971 et en 1980 à des coups d'Etat militaires. 
ruptures dans le champ politique, lui donnant par là même une possibilité de reconfiguration.

Notre compréhension de l'interaction entre le champ militant et le champ politique en Turquie et l'émergence de l'altermondialisme comme une nouvelle cause dans le champ militant est liée à ces ruptures et reconfigurations du champ politique turc.

Afin de mieux comprendre les acteurs politiques, leur interaction et l'espace des luttes dans la Turquie actuelle, il faut considérer le coup d'Etat militaire de 1980 comme un véritable point de départ.

Cette date correspond en effet à un tournant dans la composition à la fois $\mathrm{du}$ champ politique et du champ militant. Le contexte politique des années 1970 a été marqué par l'affrontement des mouvements politiques et estudiantins partisans de deux idéologies politiques bien distinctes : la gauche radicale (avec des mouvements comme Dev-Yol et Dev-Sol ${ }^{12}$ ) et l'extrême droite, connue sous le nom de «Loups gris 13 ». Déstabilisée par la montée de la gauche socialiste remettant en cause les fondements de l'Etat kémaliste, l'armée turque intervient en septembre 1980 pour mettre fin officiellement « à cette lutte inter-idéologique 14 ». Sous prétexte d'établir l'ordre et la sécurité publiques, le Parlement est dissout, tous les leaders politiques emprisonnés et interdits de vie politique active. Les activités des syndicats et des organisations professionnelles sont, elles, arrêtées.

Cette période s'est conclue par la liquidation totale des élites politiques de la gauche du pays. Des milliers d'individus ont été emprisonnés, plusieurs d'entre eux ont été exécutés. Tous les syndicats, hormis Türk-Is ${ }^{15}$ et les partis politiques ont été interdits ${ }^{16}$. La liberté d'association et de manifestation a été restreinte de

12. Dev-Yol signifie «chemin révolutionnaire » et Dev-Sol, « gauche révolutionnaire ».

13. Les «Loups gris» («Bozkurtlar» en turc) est le nom des militants des Ülkü Ocaklari («Foyers idéalistes »), mouvement d'extrême droite turc ultranationaliste. L' «Idéalisme » («Ülkücülük » en turc), idéologie développée par Alpaslan Türkes, fondateur du parti du MHP (Parti de l'action nationaliste), est un nationalisme pan-turc. Ce mouvement, très influent dans la vie politique turque, notamment dans les années 1970, face aux mouvements étudiants d'extrême-gauche, existe toujours. Ces mouvements ont tissé des liens importants avec la mafia turque, connue pour son engagement politique en leur faveur.

14. Discours du général putchiste Kenan Evren, TRT (chaîne publique nationale), 13 septembre 1980.

15 . Fondé en 1952, le Türk-Is est le premier syndicat des ouvriers en Turquie. Cependant, il est toujours resté très consensuel et proche des partis de droite centriste kémalistes. Il s'agit du seul syndicat à ne pas avoir été interdit par l'armée après le coup d'Etat.

16. Après le coup d'Etat du 12 septembre 1980, 1955 dirigeants syndicalistes appartenant au DISK (Confédération des syndicats ouvriers révolutionnaires de Turquie) sont mis en garde à vue ou torturés, 1477 civils sont jugés dans des tribunaux militaires. Pour 78 d'entre eux, la peine de mort a été recquise par le procureur. Ces personnes ont obtenu, le 24 décembre 1986, un total de 2053 ans d'emprisonnement. Le DISK et ses syndicats ont été dissous. Cela a duré jusqu'en 1991 : le 16 juillet 1991, les dirigeants du DISK sont libérés mais ils n’ont pu récupé- 
manière spectaculaire par la nouvelle Constitution ${ }^{17}$. Le coup d'Etat militaire et ses implications : lois, Constitution, pratiques gouvernementales - marque donc l'instauration d'un régime politique autoritaire ${ }^{18}$, « en contradiction croissante avec les revendications individuelles et celles $d u$ corps social : l'intérêt général est de plus en plus étranger à l'application des libertés individuelles 19 ».

Pour mieux analyser les acteurs politiques et leur interaction en Turquie, il convient également de procéder à une brève définition des acteurs et de leur positionnement dans le champ politique turc. L'arène politique est généralement occupée par certains acteurs principaux : le Parlement, le gouvernement, le président, l'armée, les syndicats, les chambres professionnelles et les organisations de la société civile. Le président joue un rôle d'arbitre, l'armée et le gouvernement, par le biais du Conseil de la sûreté nationale, délibèrent sur des décisions politiques, notamment en matière de sécurité et de politique étrangère. Cette première configuration semble désormais fragilisée à cause de la hostilité de l'armée envers le gouvernement «islamiste modéré » en place. Les syndicats et les chambres professionnelles constituent les acteurs extérieurs au champ, mais ils tentent néanmoins de participer activement aux décisions prises. Dépassant leur rôle traditionnel de défense des intérêts des adhérents, ils se retrouvent souvent impliqués dans des campagnes politiques hors de leur domaine d'activité, notamment pour la cause altermondialiste. Il existe quatre confédérations syndicales principales : Türk-Is (nationaliste), Hak-Is (conservateurs islamistes modérés), DISK (social démocrate) et KESK (socialiste-communiste), chacune étant proche d'un courant politique ${ }^{20}$. Le dernier acteur, les organisations de la société civile (voir infra), est souvent lié à l'économie, à un secteur d'activité comme TÜSIAD, représentant les hommes d'affaire en Turquie ou TOBB, l'Union des bourses, majoritairement pro-européenne. Ces organisations participent également au débat politique et jouent le rôle de groupes de pression, notamment en matière de politique d'harmonisation avec l'Union européenne. Dans ce panorama d'acteurs politiques, il n'existe pas de véritables organisations de «société civile » allant de la défense des consommateurs aux écologistes, en passant par des asso-

rer les biens de leur syndicats qu'en 1992. Pour plus d'informations, voir Güzel M.S., «Le réveil du mouvement ouvrier ", Peuples méditerranéens, n60, juin-septembre 1992.

17. Pour plus de détails, voir la loi n²908 sur les associations.

18. Bülent Tanör évalue actuellement à 800 le nombre de lois édictées dans tous les domaines par les militaires entre 1980 et 1983, n'ayant pas été soumises au contrôle ni du Parlement ni des hautes juridictions : Conseil constitutionnel ou Conseil d'Etat. Des lois aussi importantes que celles sur les partis politiques, les élections, les associations et les manifestations sont encore définies dans les termes restrictifs voulus par les militaires d'alors. Le plus curieux est sans doute qu'aucun des partis politiques ayant ensuite été au pouvoir n'a voulu ou pu modifier ces textes. Voir Bülent T., "Siyasi Tarih" (Histoire politique), in Aksin S., Bugünkü Türkiye 5. Bölüm 1980-1995 (La Turquie actuelle, 5e volume), Istanbul, 1995.

19. Groc G., « La "Société civile" turque entre politique et individu », CEMOTI, n²6, juilletdécembre 1998, pp. 43-74.

20. Respectivement, KESK (Confédération des syndicats des travailleurs publics), DISK (Confédération des syndicats des ouvriers révolutionnaires), TÜRK-IS (Confédération des syndicats des travailleurs de Turquie), HAK-IS (Confédération des syndicats réels de Turquie). 
ciations ayant un certain poids dans le champ politique ${ }^{21}$. Malgré le développement du nombre d'associations, ces dernières sont peu mobilisées dans des campagnes socio-politiques. D’après Gilles Dorronsoro, loin de se construire systématiquement contre l'Etat, les associations restent majoritairement très locales et apolitiques. Beaucoup de leurs revendications concernent l'accès à des ressources matérielles et ne récusent pas le système politique ${ }^{22}$. Des associations de type environnemental, de consommateurs, d'étudiants (notamment sous forme syndicale) sont encore novices en matière de ressources financières et politiques. Les manifestations de rue et divers mouvements sociaux sont souvent organisés sous l'égide des syndicats et des chambres professionnelles. Ces deux types de structures occupent également une place importante dans l'organisation et la diffusion du mouvement altermondialiste. Nous reviendrons sur leur rôle au moment de la présentation du mouvement.

Les années 1980 sont donc synonymes de transformation du champ politique turc. Le caractère autoritaire et sécuritaire du régime devient prépondérant avec la nouvelle Constitution approuvée en 1982. Néanmoins, nous n'observons aucune rupture dans les politiques économiques de la Turquie. Les décisions économiques du 24 janvier 1980, ratifiées avec le FMI par le gouvernement précédent présidé par Süleyman Demirel, prévoyant une politique d'austérité, ont toutes été mises en pratique par le gouvernement provisoire de l'armée après le coup d'Etat ${ }^{23}$. Après le retour à la « normalisation politique » par les élections, le gouvernement ANAP a continué à appliquer le même programme et le Premier ministre, Turgut Özal, a même réalisé la libéralisation totale des marchés dès 1990.

Alors que la libéralisation économique a continué à se développer sans connaître de grande rupture, une réelle participation politique n'a commencé à voir le jour qu'à partir des années 1990. Les interdictions et les procès contre des syndicats comme DISK s'achèvent au début de ces années. C'est aussi à partir de ce moment que nous assistons à une résurrection des mouvements sociaux. $\mathrm{Au}$ début, le mouvement ouvrier, puis les nouveaux mouvements sociaux concernant, notamment, des causes écologiques, réclament leur place à l'intérieur du champ politique. L'année 1990 est, en dépit de toutes ces évolutions, celle de l'abandon de l'économie planifiée et la libéralisation des marchés en Turquie.

En mars 1989, on assiste à une véritable explosion sociale, à un nouveau départ pour le mouvement ouvrier. Les mois de mars et d'avril 1989, appelés

21. On recense pourtant plus de 70000 associations en Turquie, d'après la base de données du ministère des Affaires intérieures :

www.dernekler.gov.tr/_Dernekler/Web/Gozlem2.aspx?sayfaNo=368

22. Dorronsoro G., "Mobilisations et régime sécuritaire ", La Turquie conteste, Paris, CNRS Editions, 2005, p. 16. L'auteur souligne également que ce constat rejoint les conclusions d'autres enquêtes sur les pays arabes.

23. Yildirim K., Türkiye Isçi Sinifi ve Sendikacilik Hareketi Taribi (L'Histoire de la classe ouvrière et du mouvement syndical de la Turquie), Istanbul, Kaynak Yayinlari, 2003, p. 195. 
"printemps du mouvement ouvrier », représentent le passage d'un mouvement muselé et meurtri à un mouvement ouvrier animé et politisé. Ce mouvement ouvrier prend l'allure d'un mouvement populaire dès février 1990. D’autres couches sociales entrent alors à leur tour en lutte. Les étudiants de différentes universités, et particulièrement celles d'Istanbul, d'Izmir et d'Ankara, organisent des forums, des manifestations et des meetings pour soutenir les grévistes et les petits producteurs lésés ${ }^{24}$.

Ces grèves sont suivies par d'autres mouvements, surtout à partir de 1994, année de crise économique en Turquie causée par la fuite des capitaux financiers. Ces mouvements de grève constituent un tournant important dans l'esprit des militants du mouvement altermondialiste. Ils sont souvent « comparés aux grèves de 1995 en France, et illustrent la résurrection des mouvements sociaux en Turquie 25 ». Ces années 1989-1995 demeurent ainsi celles où les effets des décisions des acteurs internationaux sur l'économie turque deviennent de plus en plus flagrants. Avec la politique de stabilité imposée par le FMI en 1994, on assiste à des licenciements massifs et à l'accentuation de la pauvreté. En 1996, la Turquie adhère à l'Union douanière de l'Union européenne, ce qui ouvre le marché turc à la concurrence sévère des produits européens. Tous ces événements incitent les milieux syndicaux à réfléchir sur le rôle joué par les institutions internationales (FMI, UE, etc.) dans des difficultés économiques rencontrées par la Turquie. En outre, la facilité d'entreprendre des firmes multinationales et leur protection sans limite de la part du gouvernement turc introduit dans l'agenda des mouvements sociaux la problématique de la mondialisation. Les effets de la déréglementation des marchés ne sont pas uniquement perçus dans le cadre des droits des ouvriers, mais également dans le domaine de l'environnement. Des mouvements sociaux marqués entre autres par des revendications concernant la protection de l'environnement voient ainsi le jour. L'exemple le plus représentatif de ces mobilisations est le mouvement des paysans de Bergama contre l'entreprise Eurogold, qui prévoyait l'exploitation de la mine d'or à Bergama avec l'utilisation de cyanure. Ce mouvement, créé il y a plus de dix ans, est parvenu à faire interdire à l'entreprise l'utilisation dudit produit. Au même moment, d'autres campagnes sont venues s'ajouter à cette lutte. Un mouvement antinucléaire est créé pour protester contre les projets de construction d'une centrale nucléaire à Mersin Akkuyu ${ }^{26}$. Coordonnée entre 1992 et 2000 par «La Plateforme anti-nucléaire » 27, il a réussi à faire annuler le projet. Ces mouvements de défense de l'environnement se sont encore renforcés suite à l'ouverture des bureaux de Greenpeace dans le pays

24. Ibid.

25. Entretien avec Sertug Çiçek, porte-parole de l'Antikapitalist, 9 décembre 2003, Ankara, Turquie.

26. Mersin Akkuyu est située au Sud de la Turquie, sur la côte méditerranéenne.

27. Voir Keskin M. et Keskinsoy E., "Savas Karsitliginin siyaseti : Yeni bir dünya için yeni eylemler ve Greenpeace usülü mücadele» («Politique de l'anti-guerre : nouvelles actions pour un nouveau monde et lutte à la manière de Greenpeace »), in Akinsoy O., Özen Ö., Dünyanin bütün sokaklari isyanda (Toutes les rues du monde sont en rébellion), Istanbul, Everest, 2003, pp. 339-350. 
en 1997. Actuellement, de nouveaux mouvements contre la construction de centrales nucléaires à Sinop et à Elbistan poursuivent leur campagne.

Autrement dit, le «printemps ouvrier » constitue non seulement le point de départ des mouvements sociaux, mais aussi le véritable fondement historique et symbolique des revendications altermondialistes par la suite.

Aujourd'hui, malgré la relative normalisation de la participation politique suite au coup d'Etat militaire, le champ politique turc n'est toujours pas entièrement autonome et stable, mais la candidature d'adhésion à l'Union européenne a davantage assoupli les règles du jeu. Les lois d'harmonisation ont limité le rôle des militaires dans la vie politique et, malgré leurs déclarations régulières sur les questions vitales de la politique étrangère de la Turquie (Chypre, UE, relations avec les Etats-Unis etc.), ils ne sont plus en mesure de maîtriser les autres acteurs du champ politique (gouvernement, Parlement, etc.) comme auparavant. Leur affrontement explicite avec l'AKP (Parti de la justice et du développement, islamo-conservateur modéré au pouvoir) met à mal leur popularité auprès d'un peuple dont $47 \%$ a voté en faveur de ce dernier lors des dernières élections législatives en juillet 200728.

\section{Evolution des luttes sociales et du champ militant}

Les luttes sociales ont longtemps été subordonnées aux courants politiques en Turquie. Les mouvements récents autour de la question nucléaire et écologique commencent à faire la différence dans ce domaine. Dans ce cas de figure, à la difficile autonomisation du champ politique due au poids de l'armée, se substitue une autonomisation difficile du champ militant, en raison de la dichotomie entre partis politiques et mouvements de lutte. Ces derniers ont longtemps été organisés avec le parrainage des syndicats qui, eux-mêmes, ont été chacun subordonnés à un parti politique. Les mouvements étudiants des années 1970 ont été également subordonnés, comme nous l'avons expliqué, soit à de grands courants politiques de l'extrême gauche comme Dev-Yol, Dev-Sol, ou encore le MLKP (parti marxiste-léniniste communiste), soit aux courants nationalistes comme les «Loups gris » autour du MHP (parti du mouvement nationaliste).

Aujourd'hui, cette dépendance semble s'atténuer, malgré la présence formelle ou informelle de tous ces courants politiques. L'effet générationnel n'y est pas pour rien. Le traumatisme du coup d'Etat de 1980 sur les mouvements d'extrême gauche ne permet guère à ces derniers de donner un nouveau souffle et de lancer leur courant autour d'un mouvement politique. Cependant l'héritage de ces organisations persiste, surtout pour ce qui concerne les pratiques et habitudes de l'organisation et la réalisation de l'action collective. C'est dans ce contexte que

28. Erdi Lelandais G., op. cit., p. 3. 
l'altermondialisme peut représenter un nouveau champ d'action pour toutes les luttes sociales, bien qu'il se heurte souvent à ce fonctionnement dichotomique des organisations de la gauche traditionnelle que l'on peut définir comme marxistes-léninistes, dominées par l'héritage stalinien. Le mouvement a dès le départ rencontré de nombreuses difficultés pour sortir de la structuration centralisée des organisations politiques. Il est utile de préciser que la culture politique, ou tout simplement l'expérience des mouvements sociaux et des forces politiques en Turquie, n'est pas souvent favorable à l'idée d'un espace ouvert et horizontal, au service de mouvements, de même qu'elle n'est pas favorable aux syndicats, aux ONG, aux initiatives et associations de tous types, engagés chacun à leur manière, dans leur propre secteur d'action et selon leurs méthodes ${ }^{29}$. De surcroît, les forces politiques de la gauche sont connues depuis les années 1970 pour leurs innombrables épisodes de divisions et de sous-divisions. Ainsi, en Turquie, le mouvement altermondialiste pâtit du :

«manque de coordination multisectorielle sur le plan national, qui empêche la mobilisation au niveau international, de la faiblesse de la "nouvelle gauche" face à une "gauche traditionnelle" empreinte de "souverainisme", de l'importance donnée à la notion de discipline dans des organisations politiques d'extrême gauche peu enclines à se reconnaître dans les structures flexibles du mouvement altermondialiste ${ }^{30}$. »

Toutefois, une mobilisation autour des thèmes de la guerre, de la justice globale, de l'antinucléaire, des droits démocratiques ainsi que de la contestation des OGM s'organise, malgré les difficultés que nous venons de souligner. Celle-ci a émergé en 2001, au moment des manifestations de Gênes (Italie) contre le sommet du G8. Depuis, les militants de diverses organisations ont participé à toutes les manifestations et rassemblements altermondialistes, y compris au processus de préparation des forums sociaux. La présence la plus significative des militants turcs a été observée à l'occasion du 4 e Forum social mondial à Mumbaï (Inde) en 2004, avec 80 militants, puis au 4e Forum social européen à Athènes (Grèce) en 2006, avec 1000 militants et de nombreux séminaires et ateliers animés par des organisations turques. Le mouvement a également coordonné deux manifestations importantes, l'une contre le sommet de l'OTAN en juin 2004, l'autre contre la Banque asiatique de développement en mai 2005 à Istanbul. Elles ont été organisées au niveau international, avec la participation de militants étrangers par le biais d'une liste de discussion par Internet établi à partir de janvier 200431.

29. Cité dans Baykan B.G., Erdi Lelandais G., "Cross-Readings of the anti-globalisation movement in turkey and beyond. Political culture in the making”, International Social Science Journal, $\mathrm{n}^{\circ} 182$, pp. $519-528$.

30. Gobille B., Uysal A., « Cosmopolites et enracinés », in Agrikoliansky E., Sommier I. (dirs.), Radiographie du mouvement altermondialiste, Paris, La Dispute, 2005, p. 125.

31. Entretien réalisé avec Cicek S., Antikapitalist, Ankara, avril 2004. 


\section{Spécificité de l'altermondialisme en Turquie}

La cause altermondialiste est avant tout l'œuvre de groupes politiques d'extrême gauche en Turquie et, en l'occurrence, de partis trotskystes. Ce n'est que lorsque le mouvement commence à trouver de l'écho que d'autres groupes politiques (issus de l'héritage marxiste-léniniste) décident d'y participer. Cependant, dès le départ, les représentants de certaines chambres professionnelles, dont celles des ingénieurs, des médecins, et certains syndicats s'y impliquent, en particulier autour de la contestation des firmes multinationales, du FMI et de l'OMC. On peut même dire que la première réaction altermondialiste est apparue grâce à l'implication de quelques syndicalistes de DISK ${ }^{32}$ pour créer le groupe anti-MAI, dans l'objectif de combattre l'Accord multilatéral sur l'investissement lancé en 1998 par l'OMC ${ }^{33}$.

Dans le mouvement, la présence des organisations de gauche, à la fois politiques et syndicales, est patente. Ce constat n'est pas des plus surprenants puisqu'on observe également la prédominance des organisations de gauche dans l'altermondialisme dans sa globalité. L'originalité de l'altermondialisme en Turquie vient surtout des chambres professionnelles et de la présence des associations musulmanes, en l'occurrence Mazlum-Der et Özgür-Der.

Les associations musulmanes présentes dans le mouvement n'ont pas la forme que nous pouvons retrouver en Grande-Bretagne notamment. Elles ne possèdent pas de liens transnationaux avec d'autres mouvements musulmans tels que les «Frères musulmans », mais trouvent plutôt leur source dans l'ancrage local, souvent lié aux revendications du port du voile islamique dans les universités. Ces associations sont souvent proches du parti AKP au pouvoir, bien que cette proximité semble se fragiliser devant l'hésitation du gouvernement à entreprendre les réformes demandées en faveur du port du voile ${ }^{34}$. Le gouvernement ne veut pas détériorer ses relations, déjà fragiles, avec les élites militaro-kémalistes du pays, détentrices actuelles des postes clés à responsabilité, comme les présidences des universités ou les hautes instances juridiques telles que la Cour suprême et la Cour

32. Parmi les quatre confédérations syndicales, seuls le KESK et le DISK participent activement aux campagnes altermondialistes. Dans les deux autres, seuls quelques syndicats comme Petrol-Is (syndicat des travailleurs de la pétrochimie) et Yapi-Yol-Is (syndicat des ouvriers) y figurent.

33. Ce groupe fonctionnait comme groupe d'information. Il organisait des séminaires et les réunions au sein des syndicats pour informer les travailleurs sur les méfaits de cet accord, et sur l'OMC et la mondialisation en général. Il a créé un site Internet avec tous les documents et rapports produits par le groupe : www.antimai.org. Depuis 1999, l'anti-MAI se réunit mensuellement, communique avec une liste de discussion par Internet et organise régulièrement des conférences sur des sujets précis de l'économie internationale et de la mondialisation. Le groupe apparaît dans sa dimension marxiste et socialiste. Rejetant la position réformiste d'une partie du mouvement altermondialiste, il prône un renversement total du système et des institutions actuelles et considère la mondialisation comme un des stades du capitalisme.

34. Cette situation a changé suite à la loi votée au Parlement en mars 2008, autorisant le port du voile dans l'enseignement supérieur. 
constitutionnelle. Toutefois, la place des musulmans dans l'altermondialisme turc est indéniable. Ces derniers sont souvent aux côtés des organisations d'extrême gauche dans les campagnes, ils ont notamment entrepris une campagne d'action sur les conditions de détention des prisonniers politiques, majoritairement de gauche ${ }^{35}$. Ils ont également participé à l'organisation des assemblées préparatoires du Forum social européen (FSE) à Istanbul en avril 2005. Comment pouvons-nous expliquer leur place dans l'altermondialisme?

Celle-ci est liée à une tendance de fond des courants religieux, lesquels, dans un contexte "laïc», ont toujours pratiqué une culture de la transversalité, contournant d'abord par des réseaux discrets la pression des autorités, mais accomplissant aussi dans des regroupements associatifs des activités traditionnelles de solidarité conformes aux préceptes religieux ${ }^{36}$. De plus, la critique de l'autoritarisme d'Etat, surtout lorsque celui-ci est laïc, la contestation, la mobilisation de la base, voire une argumentation d'antagonisme entre l'Etat et la société, ne leur sont pas étrangères. Mais c'est à partir des années 1980, à la faveur de tels changements de perspective, que des thématiques civiles ont commencé à intéresser les milieux religieux, comme les questions des droits de l'Homme et de la démocratie dans le cas de Mazlum-Der et d'Özgür-Der ${ }^{37}$. Cela s'est fait avec d'autant plus d'écho que des contacts ont été pris dès le milieu des années 1980 avec des intellectuels de tous bords, dans l'intérêt nouveau - mais commun à tous - d'inventorier des questions telles que les dimensions sociologiques de l'action politique, une approche comparative des modalités de la démocratie, de la citoyenneté, l'importance des référents universels et la mondialisation ${ }^{38}$.

En Turquie, l'islamisme, présent dans le système politique mais tenu sous étroite surveillance, génère une contestation qui remet en cause l'hégémonie d'une laïcité perçue comme étant trop restrictive en termes de liberté de l'individu (par exemple au sujet de l'interdiction du port du foulard des jeunes filles à l'université) ${ }^{39}$. Les milieux de gauche et une partie plutôt modérée du milieu islamiste arrivent de ce fait à s'associer dans le cadre de certaines campagnes face à un pouvoir oppressant pour les deux parties.

Considérant la transformation de l'économie et de l'Etat selon les conditions de la mondialisation néo-libérale, les forces sociales de la périphérie doivent soit s'organiser, soit disparaitre. Originellement prévus pour fournir une

35. Pour plus d'informations sur ce sujet, voir Erdi Lelandais G., "Cosmopolites enracinés ». La transnationalité et la construction identitaire du mouvement altermondialiste : l'exemple de la Turquie, thèse de doctorat non publiée, EHESS, 2006.

36. Groc G., op. cit., p. 63.

37. Pour la représentation de ces organisations, voir le schéma en annexe de cet article.

38. Ibid.

39. Voir la conclusion de F. Khosrokhavar, CEMOTI, n²6, 1998, pp. 235-241. 
aide autonome à des populations défavorisées, les nouveaux mouvements islamiques sont devenus une source de résistance et d'alternative au matérialisme. Ainsi, le domaine traditionnel des services publics sociaux est parfois incarné par des foyers privés et des cliniques d'associations musulmanes ${ }^{40}$. Dans ce cadre, l'islam n'est pas simplement un ensemble de rites religieux, mais aussi un ciment social qui relie les «communautés » abandonnées par l'Etat néolibéral ${ }^{41}$. Cette place de l'islam provient également du fait qu'une grande partie de la société turque ait une perception religieuse d'elle-même et de son environnement. Le rôle attribué ainsi aux associations musulmanes leur permet de trouver une place dans l'altermondialisme. Elles s'affirment ainsi comme porte-parole d'une autre société, « une autre Turquie » et « un autre monde ", bien que les revendications de cet autre monde ne convergent pas toujours avec les autres organisations du mouvement altermondialiste.

Mustafa Ercan explique de la façon suivante la place de Mazlum-Der dans le mouvement altermondialiste :

\begin{abstract}
« Nous ne connaissons pas très bien les structures de ce mouvement, mais nous le considérons comme une réaction de la conscience humaine aux effets négatifs de la mondialisation. Dans ce sens-là, tout le temps que nous croyons à la justesse de l'action de ce mouvement, nous y participons. Nous n'avons pas de barrières strictes, tout mouvement ou action pour le bien de l'humanité est le bienvenu pour nous. C'est pour cela que nous trouvons nécessaire de contribuer à ce mouvement avec nos propres messages ${ }^{42}$. »
\end{abstract}

Özgür-Der adhère également à cette ligne de conduite, mais souligne davantage l'identité musulmane dans ses activités. On observe en effet dans ses brochures et sur son site Internet un nombre plus conséquent de citations tirées du Coran.

Levent Sensever considère la participation de ces deux organisations comme un phénomène important pour le mouvement : «Leur participation était particulièrement importante parce que cela a permis d'intégrer les milieux islamiques civils et politiques ayant une place considérable en Turquie dans le mouvement altermondialiste $43 »$.

Quant à la place des chambres professionnelles, leur participation constitue une des pierres angulaires du mouvement altermondialiste, une condition sine qua non de sa réussite :

40. L'exemple qui illustre le mieux cette situation est l'association Denizfeneri qui distribue des sacs de nourriture pendant le Ramadan, fournit des vêtements pour les enfants et trouve des emplois. 41. Pasha M.K., "Globalization, Islam and resistance”, Gills Barry K. (ed.), Globalization and the Politics of Resistance, New York, Palgrave, 2001, p. 250.

42 . Entretien avec Ercan M., le 12 avril 2005, Istanbul.

43. Sensever L., Dünya Sosyal Forumu (Forum social mondial), Istanbul, Metis, 2003, p. 101. 
"Quand vous réussissez à convaincre les syndicats et les chambres professionnelles pour une campagne, vous avez déjà un sentiment de sécurité. Quand vous avez ces institutions derrière vous, vous vous lancez plus facilement, avec plus de confiance, pour convaincre les autres. Les gens regardent d'abord qui a signé, s'ils ne voient pas les organisations dont ils sont sympathisants, même si le sujet les intéresse, ils ne font pas confiance. A ce moment-là, il est très difficile de mener ou réussir la campagne ${ }^{44}$. »

Dans ce cadre, l'Union des chambres des ingénieurs et des architectes de Turquie (TMMOB) ${ }^{45}$ et l'Union des médecins (TTB) sont les acteurs les plus présents dans les activités altermondialistes. Si le TTB ${ }^{46}$ ne se présente très souvent qu'avec son nom et sa signature ${ }^{47}$, c'est réellement le TMMOB qui fournit une base logistique au mouvement. Nous verrons plus loin les raisons de la présence de cette union, mais il convient tout de même ici d'évoquer le rôle que les ingénieurs s'attribuent historiquement en Turquie. Ces derniers, au-delà de leur place dans le processus de production, furent les défenseurs des modèles sociaux de développement. D’après Nilüfer Göle :

"l'idéologie nommée "social engineering”, encore applicable pour les ingénieurs de la Turquie, défend l’idée que la rationalité menée par les ingénieurs au niveau de la production peut s'étendre à la société. De ce fait, elle souligne la lutte entre le capitalisme et la civilisation industrielle plutôt que leur réconciliation. En définitive, les ingénieurs de gauche et ceux proches des milieux islamiques ont en commun de critiquer le capitalisme et de se référer au mythe de l'industrialisation ${ }^{48}$. »

Ce qu'illustrent les propos de l'ex-président du TMMOB, Kaya Güvenç :

«Les ingénieurs et les architectes en Turquie sont numériquement autour de 450000 et la plupart d'entre eux ont de bas salaires. Dans

44. Entretien avec Sertug Çiçek, Antikapitalist, 9 décembre 2003, Ankara.

45 . Fondé en 1954, le TMMOB a un statut d' "institution professionnelle à caractère public » réglementée par la Constitution turque. Les chambres professionnelles sont donc des institutions publiques avec une gestion complètement autonome par rapport à l'Etat. Pour exercer le métier d'ingénieur, il est obligatoire en Turquie de s'inscrire à sa chambre professionnelle.

46. Fondée en 1953 à Istanbul, le TTB est une organisation professionnelle à caractère public. Pour exercer le métier de médecin libéral, il est obligatoire en Turquie d'adhérer à cette organisation. Tout comme pour le TMMOB, la direction du TTB a une position souvent critique envers le régime politique.

47. Il arrive quand même parfois que son président, Gencay Gürsoy, participe au nom de son organisation à certaines campagnes et protestations du Küresel BAK, coalition de diverses organisations pour lutter contre la guerre en Irak (pour la description de cette organisation, voir l'annexe).

48. Göle N., Mühendisler ve ideoloji (Les Ingénieurs et l’idéologie), Istanbul, Metis, 1998, p. 11. 
un contexte où il n'y a plus d'investissement et pas suffisamment de ressources publiques consacrées à la recherche scientifique et technologique, les organisations des ingénieurs en sont ainsi inévitablement arrivées à une position différente. C'est pour cela qu'ils sont opposés à la mondialisation ou à l'AMI, parce qu'ils estiment que tous leurs problèmes sont le reflet de la mondialisation en Turquie. C'est pour cela aussi que notre organisation se voit obligée de s'intéresser aux macro-politiques tout en sortant parfois de son véritable domaine d'activité 49 ».

Selon Tanil Bora, la politisation intensive des années 1970 a affecté directement ces organisations professionnelles qui sont peu à peu devenues les acteurs de la politisation en Turquie. Le TMMOB, qui se trouvait dans l'aile gauche, a remarquablement contribué à la formation, à l'organisation et à la diffusion de l'idéologie de gauche ${ }^{50}$. La raison principale de cette tendance était la possibilité de relier le caractère positiviste, progressiste et développementaliste de ce groupe professionnel aux courants socialistes de l'époque. D’après Kaya Güvenç, "les ingénieurs, à partir des années 1960, se sont rendus compte du parallélisme entre leurs propres problèmes et ceux de la classe ouvrière. En raison de cela, leur opposition aux politiques de l'époque s'est accentuée 51 ». Dans les années 1960-1970, la motivation principale de ce lien était l'anti-impérialisme ${ }^{52}$. Il n'est pas anodin de constater que ce motif a continué à motiver les actions du TMMOB jusqu'à nos jours.

Dans les années 1970, les actions des ingénieurs se transforment donc en une lutte révolutionnaire marxiste-léniniste, reléguant au second plan le caractère professionnel de l'organisation des années 1950 53. Dans son rapport d'activité de 1974, l'Union souligne l'importance de «la lutte pour la démocratie de la classe ouvrière et de la résistance face aux attaques fascistes contre les ouvriers 54 ».

Le caractère combatif du TMMOB face à l'ordre établi lui a causé de nombreux ennuis durant les années 1970 et 1980, et la plupart de ses dirigeants a été écrouée. Le contrôle de l'Etat sur ses activités s'est ainsi renforcé dans le courant des années 1980, mais le TMMOB a poursuivi ses activités dissidentes avec le système politique turc. La re-politisation du TMMOB n'a cessé de croître au cours des années 1990, parallèlement au développement des demandes de démocratisation et des mouvements sociaux en Turquie ${ }^{55}$. Pendant ces années, on observe au

50. Bora T., « Türkiye’de meslek kuruluslari : "Kamu”, "Sivil" ve "Milli” nin muglak kesisim alani » (Institutions professionnelles en Turquie : espace d'intersection ambigu du public, du civil et du national), Türkiye'de Sivil Toplum ve Milliyetçilik, Istanbul, Iletisim, 2002, pp. 265-308.

51. Entretien avec Kaya Güvenç, 2 décembre 2003, Ankara.

52. Bora T., op. cit.

53. Göle N., op. cit., p. 117.

54 . Rapport d'activité de 1974 du TMMOB, p. 52.

55 . Bora T., op. cit., p. 279. 
sein du TMMOB un grand intérêt pour la critique des politiques néo-libérales et des privatisations en Turquie. Dans ce cadre, vers la fin des années 1990, la mondialisation fait son apparition dans son discours. Dans le rapport d'activité de son $35^{\mathrm{e}}$ congrès, il définit le «nouvel ordre mondial » comme la forme actuelle de l'impérialisme. Toujours d'après ce rapport, les caractéristiques principales de cet ordre sont "l'organisation de la production nationale selon les conditions et les demandes du marché extérieur », "l'activité de blocage par des instances de décision étrangères » et «la dépendance envers les pays riches concernant les aides ». Le rapport fait également allusion à une néo-colonisation de la Turquie ${ }^{56}$. Le fait de s'intéresser à l'altermondialisme provient de sa position face à la mondialisation jugée « entièrement capitaliste, inacceptable et inutile pour la Turquie, sa population et ses travailleurs 57 ». Dans cette perspective, le TMMOB a envoyé des délégués à Prague en 2000 pour se joindre aux protestations contre la réunion du FMI et a coordonné la venue des militants d'autres organisations. Néanmoins, il semble nécessaire de souligner que cette participation ne devra pas être généralisée à l'ensemble des ingénieurs membres de cette organisation. Seuls les médecins, les ingénieurs qui s'intéressent à cette cause et qui luttent pour la direction de la chambre ont pris part au mouvement.

Au-delà du rôle joué par les membres actifs des chambres professionnelles, unique en son genre quand nous considérons l'ensemble des mouvements altermondialistes au niveau européen, et outre la présence des organisations musulmanes, l'altermondialisme en Turquie se démarque également par l'omniprésence des idéologies politiques. Contrairement à l'altermondialisme global qui souligne constamment sa distance avec les partis politiques et qui fait tout, notamment à l'occasion des forums sociaux, pour s'en démarquer dans l'idéal de garder son indépendance vis-à-vis des courants politiques, le mouvement altermondialiste turc fait le maximum pour que les partis politiques fassent partie des principaux acteurs du processus. Les partis ou groupes politiques défendant dans leur majorité le socialisme, dans sa forme soit trotskyste soit marxiste-léniniste orthodoxe, participent activement aux campagnes altermondialistes. Il ne serait pas faux de défendre l'idée selon laquelle sans la persévérance des militants trotskystes, par exemple, l'altermondialisme en Turquie ne pourrait pas réaliser autant de campagnes. De plus, différentes fractions du trotskisme y sont présentes, aussi bien celle de la $4 \mathrm{e}$ Internationale par le biais de l'ÖDP que celle du courant socialiste international de Tony Cliff, par le biais du DSIP ou l'Antikapitalist. Pour eux, le mouvement altermondialiste représente un nouvel espoir, un nouveau débouché dans la réalisation du socialisme international et de la révolution permanente ${ }^{58}$. Suivant le courant international auquel ils sont rattachés, les militants de ces deux grou-

56. Rapport d'activité de la 34e mandature (1996-1998), TMMOB, p. 8.

57. Entretien avec Güvenç K., 2 décembre 2003, Ankara.

58. Pour plus d'informations sur les courants trotskystes, voir Bensaïd D., Les Trotskysmes, Paris, PUF, 2003. 
pes consacrent une énergie considérable à l'altermondialisme. Un militant explique leur implication et leur motivation de la manière suivante :

\begin{abstract}
«A partir de Prague, nous avons adopté la tactique de nos groupes frères participant au mouvement. Les gens qui venaient aux manifestations voulaient tout de suite obtenir quelque chose. Notre objectif est devenu de réunir les gens autour de points communs sans cacher notre identité, mais sans les obliger à participer au parti. Nous pensions que cela pouvait arriver naturellement dans le cours des choses 59 ».
\end{abstract}

Cette politique leur assure une certaine visibilité. En outre, les liens maintenus avec l'étranger, notamment avec le Socialist Workers Party (SWP) anglais, leur fournissent des ressources en termes de financement mais aussi d'expériences militantes. Grâce à cet appui, les militants des deux groupes vont à l'étranger et participent chaque année à une semaine de formation à Londres. L'altermondialisme devient un champ d'investissement prioritaire pour un certain nombre de militants trotskystes et cela, dès les tous premiers événements ${ }^{60}$. A travers leur militantisme et leur participation à différentes mobilisations, les militants trotskystes ont acquis un certain type de ressources utiles à la construction d'un mouvement : une insertion dans un grand nombre de réseaux (associatifs, syndicaux, internationaux, etc.) et la maîtrise de diverses formes d'action (manifestations, campagnes, actions « coup de poing ", services d'ordre, etc.) ${ }^{61}$. Cela leur donne une place non négligeable dans la construction et l'orientation du mouvement en Turquie, comme l'explique cette journaliste :

«Depuis le début, en Turquie, dans la structure formée autour du FSE, il y a un poids indiscutable des personnes et des organisations connues comme trotskystes. Elles assurent un appui intellectuel, se nourrissent du mouvement anglais. Mais il serait erroné de les résumer au courant anglais. Après la chute du Mur, ils ont affirmé avoir eu raison face à la gauche radicale traditionnelle concernant l'Union soviétique. Du coup, ils ont estimé que c'était à eux d'être à la tête d'un mouvement. A mon avis, ils ont un peu raison. Je ne suis pas trotskyste mais leur orientation me paraît cohérente 62 ».

Dans ce cadre, le militantisme des trotskystes se rapproche de la lutte symbolique, au sens de Bourdieu, pour la production du sens commun ou,

59. Entretien avec K. Basak, DSIP, 3 décembre 2003, Ankara.

60. Il s'agit des premières manifestations altermondialistes à Seattle contre l'OMC en 1999 et à Prague contre le FMI en 2000. Ces deux organisations ont monté des collectifs pour aller à Prague en 2000.

61. Johsua F., Du Cleuziou Y. R., "Entre radicalité et apolitisme ", in Agrikoliansky E., Sommier I., op. cit., p. 253.

62 . Entretien avec Engin A., journaliste, 15 décembre 2003, Istanbul. 
plus précisément, pour le monopole de la nomination légitime comme imposition officielle de la vision légitime du monde social, les agents engageant le capital symbolique qu'ils ont acquis dans les luttes antérieures 63 .

\section{Relations inter-organisationnelles dans le mouvement}

Au-delà du nouveau champ d'action que propose l'altermondialisme pour divers groupes leur permettant de continuer leur activité politique par le biais d'un mouvement social, le processus de construction des alliances demeure parfois compliqué à gérer.

Pour comprendre les relations inter-organisationnelles du système d'alliances en Turquie, il convient d'analyser la manière dont les organisations se regroupent pour constituer un mouvement, celles dont les alliances fonctionnent, ainsi que les points de convergence permettant d'entretenir ces alliances.

Compte tenu de la présence imposante des idéologies politiques au sein des organisations, le fait de travailler ensemble pour organiser un projet commun s'avère toujours ardue. Dans cette optique, les plateformes, coalitions ou coordinations sont considérées comme le meilleur moyen d'attirer un grand nombre d'organisations et de militants. Ces types d'organisations permettent en effet à chaque composante du mouvement de préserver sa liberté et de contribuer, selon sa propre volonté, à la campagne. Elles donnent l'image de formes de représentation "démocratiques ", permettant la libre expression des acteurs sociaux mobilisés ${ }^{64}$. En Turquie, il paraît plus aisé de convaincre les organisations de participer à une coordination ou à une plateforme. En effet, étant donné la confiance inter-organisationnelle limitée, il s'avère que beaucoup d'organisations se méfient des « organisations parapluie » du type ATTAC regroupant plusieurs organisations convergentes sur certaines revendications précises, par crainte de s'y fondre et de perdre leur propre pouvoir. Or, les coordinations et plateformes, par leur souplesse, permettent à toute organisation de quitter la structure à n'importe quel moment. De plus, elles fournissent une certaine garantie aux personnes non organisées craignant d'être influencées par des organisations politiques, ainsi qu'un espace de liberté au sein duquel les non-organisés ont également un droit de parole, à côté des direction des organisations. C'est le caractère opérationnel donc leur caractère opérationnel qui est plébiscité :

«Le mouvement essaie d'avancer par des plateformes. Avant, nous n'avions aucune tradition de travailler ensemble. Nous avions peutêtre la possibilité de rencontrer DSIP ou Antikapitalist, ou de lire

63 . Bourdieu P., op. cit., p. 307.

64 . Hassenteufel P., «Pratiques représentatives et construction identitaire. Une approche des coordinations ", Revue française de science politique, ${ }^{\circ} 1$, vol. 41, 1991, pp. 5-27. 
leur revue, mais c'était impossible avec d'autres organisations. La Coordination contre la guerre fut une très bonne organisation. Plus de 180 organisations ont donné leur signature pour la coordination. Son efficacité était évidente, prenant ses informations du mouvement international. Du coup, elle nourrissait le mouvement très correctement. C'était une structure, les individus comme les organisations pouvaient s'exprimer librement. Malgré le poids des organisations, les individus n'étaient pas exclus 65 ».

Les coordinations et les plateformes se distinguent également par leur existence brève, parfois limitée à la durée du conflit même auquel elles sont liées, et par une volonté d'auto-contrôle de la lutte par ceux qui la mènent. C'est pour cette raison qu'il est souvent question de "mouvements identitaires limités » lorsqu'il s'agit de coordinations 66. La " Plateforme anti-guerre », créée au lendemain du lancement des opérations militaires en Afghanistan, s'est transformée en "Coordination contre la guerre en Irak ». Une fois l'auto-contrôle des militants affaibli dans la coordination, celle-ci s'est divisée et séparée d'une partie de ses membres.

Cependant, les jalons de ce type d'organisation s'avèrent fragiles pour maintenir les alliances construites. Tout d'abord, il est toujours nécessaire de trouver un sujet susceptible de motiver un grand nombre de personnes malgré l'expression de sensibilités différentes. Par ailleurs, la vigilance est de mise pour maintenir le sujet au centre des préoccupations de l'ensemble des participants, sans qu'il y ait de dispersions vers d'autres sujets potentiellement moins attractifs en termes d'engagement et de soutien pour certaines des composantes. Ces deux conditions ont été réunies par la Coordination, ce qui explique sûrement le fait qu'elle ait réuni autant d'acteurs, le choix de la guerre comme sujet de lutte était motivant pour tous les participants : «Du côté des organisations d'extrême gauche, leur position anti-guerre s'expliquait en termes de lutte contre une guerre impérialiste. Pour les islamistes c'était en terme de fraternité musulmane 67 ». Le point commun était la mobilisation contre la guerre et l'arrêt de celle-ci, ce qui a été suffisant pour la collaboration de ces deux groupes dans la Coordination. Enfin, comme l'a souligné un militant, un autre problème réside dans le fait de faire partager ce processus aux grandes organisations politiques et syndicales :

«Les coordinations se créent d'abord au niveau des institutions. La raison est que les institutions comme TMMOB ou KESK sont tièdes avec des processus se développant en dehors d'elles. Elles ont

65. Entretien avec Ömeroglu P., Union internationale des travailleurs, Yapi-Yol Sen, le 8 décembre 2003, Ankara.

66. Hassenteufel P., op. cit., p. 5.

67. Entretien avec A. Engin journaliste, 15 décembre 2003, Istanbul. 
leur propre façon de travailler [...]. Le processus de les convaincre sans les effrayer est donc difficile. Ce sont de grandes organisations de masse dans lesquelles de nombreuses alliances, des individus de divers courants politiques existent 68 ».

A cela s'ajoutent les phénomènes de marginalisation / monopolisation et les stratégies de maîtrise du réseau. Dans ce domaine, un effort considérable a été fait par le DSIP. Le Forum social d'Istanbul (FSI) ${ }^{69}$ a été créé à l'initiative des militants du DSIP. Nous constatons un contrôle très fort de cette organisation sur le Küresel BAK et le FSI. Si, en principe, ces deux organisations sont ouvertes à la participation de tous, une très forte logique de sélection existe. Dans les réunions de travail du FSI auxquelles nous avons participé, nous avons certes observé une diversité quant au profil (politique) des participants (Verts, indépendants, non-organisés, ÖDP, sociaux-démocrates, musulmans, etc.) mais nous avons également pu noter l'absence de certaines organisations. En effet, aucune possibilité de contribution de l'Antikapitalist n'est envisageable dans une structure comprenant le DSIP, compte tenu de la rivalité qui oppose ces deux organisations, dans leur volonté d'être les uniques représentants du courant socialiste international en Turquie. Dans ce cadre, le DSIP tente régulièrement de marginaliser l'Antikapitalist, numériquement plus faible.

Les stratégies de sélection et de leadership que nous observons davantage parmi les organisations de la gauche socialiste au sein du mouvement ont également été employées vis-à-vis des organisations musulmanes. Un parti de cette gauche ancrée dans une tradition républicaine et läque a longtemps rechigné à les accepter, en raison de leurs revendications sur le voile islamique. C'était notamment la position du TMMOB et d'une partie de l'ÖDP. Mais ce clivage semble désormais dépassé.

Récemment, la place du mouvement kurde dans l'altermondialisme a posé certains problèmes, bien que tout le monde se soit accordé sur l'indispensable présence des Kurdes dans un mouvement comme l'altermondialisme, cette participation dépendait tout de même de certaines conditions implicites. Le fait que le DTP et ses militants aient préparé, pendant la journée d'action globale contre la guerre le 15 février 2003, des slogans pour la libération d'Abdullah Öcalan (leader emprisonné du mouvement kurde), a porté un coup fatal à leurs relations avec d'autres organisations de gauche, notamment avec le DSIP et l'ÖDP. L'intégration explicite des revendications kurdes dans le mouvement antiguerre n'était pas souhaitée par ces organisations, qui craignaient la perte de crédibilité et de prestige du mouvement aux yeux de l'opinion publique.

68. Entretien avec L. Sensever, DSIP et FSI, 29 mars 2005, Istanbul.

69. Pour la description du forum, voir l'annexe de cet article. 


\section{Altermondialisme : transformation du militantisme et la « réma- nence ${ }^{70}$ " politique}

L'altermondialisme en tant que revendication est incontestablement nouveau dans le champ militant turc. Les sujets qu'il défend, l'organisation des campagnes, la souplesse et l'horizontalité des structures, l'absence de hiérarchie parmi les militants fait de ce mouvement en Turquie un espace qui reste entièrement à découvrir et avec lequel les organisations jusqu'alors détentrices du monopole de la contestation, arc-boutées sur leurs structures rigides ainsi que les militants fidèles doivent désormais composer.

Il est vrai que le débat sur l'évolution du militantisme est vif. S'agit-il de la transformation du militantisme traditionnel ? Peut-on parler de la fin du militantisme « sacrifice » 71 ? Voici tout un ensemble de questions auxquelles il n'est pas aisé de répondre. Si on peut s'accorder sur l'affaiblissement des grandes organisations politiques de la gauche radicale comme le parti communiste, pouvons-nous pour autant parler de la « fin des militants » ? Jacques Ion avance, dans son ouvrage Militer aujourd'hui, l'idée qu'il est désormais possible d'observer un engagement distancié satisfaisant enfin les aspirations à l'autonomie individuelle des nouveaux militants ${ }^{72}$. D'autres, comme Johanna Siméant, critiquent cette vision et dénoncent l'approche opposant le militantisme partisan au militantisme associatif 73 . Concernant la Turquie, les deux thèses semblent, selon nous, valables. On peut même avancer l'idée que l'altermondialisme, avec d'un côté ses connotations fortement politiques en raison de la participation active de partis et, de l'autre, la flexibilité de son organisation, propose un engagement hybride. Autrement dit, il propose à la fois une forme d'émancipation pour certains militants d'organisations partisanes et une forme de rémanence politique pour d'anciens militants et courants politiques par le biais de structures associatives, de coordinations et de coalitions. Il permet également aux individus méfiants envers les organisations politiques de militer sur des sujets politiques et sociaux sans subir la discipline de ces dernières, mais tout en profitant de leurs ressources de mobilisation.

Cela étant, le militantisme en Turquie, longtemps dominé par une forme de «militantisme sacrifice ", subit une transformation sous les effets du mouvement altermondialiste et de son fonctionnement fluide. Ce fonctionnement

70. Taylor V., « La continuité des mouvements sociaux. La mise en veille du mouvement des femmes », in Fillieule O. (dir.), Devenir militants, sociologie du désengagement militant, Paris, Belin, 2005.

71. Pudal B., Prendre parti. Pour une sociologie historique du PCF, Paris, Presses de Sciences Po, 1989. Pudal utilise ce terme pour désigner les militants fidèles à l'orientation politique de leur organisation sans jamais la mettre en cause, et qui se consacrent uniquement à la réussite et l'expansion de ses idées.

72 . Ion J., Franguiadakis S., Viot P. (dirs.), Militer aujourd'bui, Paris, Cevipof, Autrement, 2005.

73. Siméant J., «Un humanitaire apolitique ? Démarcation, socialisation au politique et espaces de réalisation de soi », in Lagroye J., La Politisation, Paris, Belin, 2003, pp. 163-196. 
assure non seulement une relation souple entre l'individu et son organisation, mais permet aussi aux individus d'affirmer leur identité et d'exprimer leur vision du monde, libérés de leur appartenance politique. Ainsi, l'individu est moins contraint par les normes imposées par l'organisation à laquelle il appartient, quand bien même son identité politique continuerait par ailleurs à déterminer ses actes. Comme l'observe Kara Umut, militant anarchiste vert :

«Avant mon implication dans l'anti-mondialisation, j'étais un stalinien pur et dur, j'ai milité aussi dans le PCT [parti communiste turc]. Je me voyais comme guerrier de la lutte des classes. Après, j'ai travaillé pour la création de la fédération anarchiste. Mais j'ai vu que dans les relations interpersonnelles et les principes communs, il y avait une sorte d'obligation pour les individus. Il fallait se comporter selon les règles de l'organisation, on n'avait pas le droit de discuter les décisions de la direction. Soit tu acceptais, soit tu étais exclu. En raison de ce fonctionnement, j'ai quitté la Fédération. Je me vois mieux dans ce type de militantisme. Nous sommes proches des Black Blocs $\left.{ }^{74}\right]$, nous n'essayons pas de garder l'organisation débout. Notre groupe est basé sur des relations volontaires, il est autonome et peut être dissout à tout moment. C'est un réseau où il n’y a pas de dirigeants ni un seul porte-parole ${ }^{75}$ ».

Un nouveau rapport au militantisme semble donc se dessiner, proche du concept d' « engagement distancié » proposé par Jacques Ion, un rapport marqué par une volonté de maîtrise de l'autonomie des convictions individuelles par rapport aux prises de positions ou directives organisationnelles ${ }^{76}$. Si l'engagement militant est motivé par la nécessité de résister, un certain hédonisme s'affirme. L'action collective n'est pas uniquement perçue comme une œuvre libératrice dans ses objectifs, mais également par elle-même, avec des effets immédiats pour l'individu. Cette évolution demande une intensification de l'engagement individuel et le déclin de la figure du «petit soldat derrière ses chefs » au profit de la participation. Ce passage d'un militantisme de masse à un militantisme d'acteurs correspond à une nouvelle façon d'être-ensemble qui favorise l'inclusion. La manifestation devient alors un espace ouvert à la contribution de chacun, sans autres pré-requis que le partage de ses objectifs immédiats. Mais cette souplesse de fonctionnement se heurte parfois au blocage de grandes organisations.

74. Il s'agit des groupes proches des anarchistes, organisés durant des manifestations pour réaliser des actions directes. Pour une analyse détaillé de ces groupes, voir Dupuis-Deri F., «Penser l'action directe des Black Blocs », Politix, vol.17, n68, 2004, pp. 79-109.

75 . Entretien avec Kara Umut, anarchiste vert, 18 novembre 2003, Istanbul.

76. Mathieu L., "Les nouvelles formes de la contestation sociale », Regards sur l'actualité, n²51, mai 1999, pp. 33-44. 
«Notre mouvement a une autre forme de fonctionnement. Les directions des grandes structures comme TMMOB, DISK ou KESK ont du mal à accepter de se retrouver avec de simples individus autour de la même table. Mais c'est comme ça. Il est clair qu'on ne peut pas faire sans ces organisations, mais le mouvement ne peut pas faire non plus sans une base de militantisme individuel. C'est aussi le fondement de la démocratie participative ${ }^{77}$. »

Chez certains, le militantisme dans le mouvement se substitue à l'engagement partisan, au terme d'un réexamen implacable de leurs trajectoires militantes antérieures et d'une critique sévère vis-à-vis du champ politique ${ }^{78}$, Comme l'explique Kemal Basak, un militant du DSIP :

«A partir de notre implication dans le mouvement, la campagne, c'està-dire le fait de sensibiliser les individus autour de vous et de les convaincre de participer à la manifestation, est devenue plus importante. Elle est devenue plus attrayante pour certains camarades. Ils ont oublié l'organisation et se sont laissés influencer par d'autres courants dans le monde. Quand on est allé au sommet alternatif à Thessalonique, une partie des militants l'a interprété complètement différemment. Pour eux, le sommet était organisé par des groupes autonomes composés chacun de quelques personnes sans qu'il y ait un mécanisme au dessus d'eux. Ce fonctionnement les a attirés. Ils ont affirmé que le mouvement altermondialiste permettait même aux simples individus d'organiser une action. Du coup, ils ont quitté le DSIP. Ils croyaient ne pas avoir besoin d'une organisation pour militer ${ }^{79}$. »

Cela correspond en effet au pragmatisme de l'action ainsi qu'à l'activisme qui prémuniraient certains militants contre les querelles idéologiques. Ce type de militantisme se rapproche du concept de "militantisme affranchi », qui porte en lui la volonté de préserver son quant-à-soi 80.

Cependant, la transformation du militantisme en Turquie que nous venons de décrire n'est pas non plus une évolution qui pourrait véritablement bousculer les fondements du militantisme politique dans le pays. Malgré la mise en avant de plus en plus flagrante du militantisme individuel, le rôle et le poids des organisations politiques perdurent, d'autant plus qu'au sein de l'altermondialisme, ces organisations politiques se retrouvent avec leur base militante et constituent une condition de réussite des campagnes. Par rapport au passé, les

77. Entretien avec Mater T., porte-parole du Küresel BAK, 11 décembre 2003, Istanbul.

78. Sommier I., Les Nouveaux mouvements contestataires à l'heure de la mondialisation, Paris, Flammarion, 2001, p. 52.

79. Entretien avec K. Basak, DSIP, 3 décembre 2003, Ankara.

80. Ion J., Franguiadakis S., Viot P. (dirs.), op. cit., p. 82. 
membres de ces organisations préfèrent l'altermondialisme pour militer, car la répression étatique est moins directe s'agissant des mouvements associatifs à revendications internationales. Ce type de militantisme s'effectue surtout via la rémanence politique d'anciens militants d'extrême gauche qui voient dans l'altermondialisme la nouvelle cause politique à défendre. Certaines fractions de militants ont ainsi trouvé dans l'altermondialisme la possibilité de reconversion de leurs anciennes luttes. C'est particulièrement le cas des groupes trotskystes. Peu à peu intégrés à cette logique, les groupes communistes de tradition stalinienne ont également converti une partie de leurs revendications dans le mouvement altermondialiste, malgré leur résistance initiale pour conserver les structurations politiques traditionnelles de leurs groupes.

Le mouvement altermondialiste semble donc avoir joué un rôle de « rémanence ", en assurant le maintien de certaines idées, d'une identité collective, ainsi que la survie de réseaux d'activistes riches en ressources et utiles pour de nouvelles mobilisations. Le mouvement fournit de ce fait aux groupes d'extrême gauche des cadres de réinvestissement militant privilégiés, en procurant aux partis politiques de cette orientation une possibilité de «blanchiment » de certaines thématiques très marquées à gauche, voire " marxisantes ", un nouveau cadrage moins connoté politiquement, mais permettant de continuer à faire avancer un ensemble de revendications propres à ce courant 81 .

On peut également voir dans cet investissement le reflet des mutations qui ont affecté le champ de la gauche extra-parlementaire au cours de la décennie 1990. Il est le résultat à la fois de choix stratégiques de la part d'organisations (en l'occurrence le DSIP), mais aussi et surtout de décisions individuelles ou de très petits groupes, souvent par défaut : individus déçus par leur groupe d'appartenance d'origine ou orphelins de leur organisation, notamment pour le groupe Antikapitalist exclu du DSIP en 1997 par exemple ${ }^{82}$. Le fait d'observer la présence dominante des militants de longue date dans le mouvement vient confirmer ce constat. Dans l'altermondialisme turc, un profil militant assez particulier se dessine. Celui-ci concerne des militants très marqués à gauche, qui participent à toutes les campagnes altermondialistes tout en affichant des priorités diverses. Les résultats de nos entretiens avec ces militants nous ont montré qu'en réalité, ces individus ont un passé de militantisme intense, qu'ils ont été membres d'organisations politiques étudiantes dans les années 1970 et qu'ils ont subi des arrestations policières et, souvent, des faits de torture ${ }^{83}$. Parmi eux, certains ont fait de la prison au lendemain du coup d'Etat de 1980, soit en raison de leur appartenance aux groupes d'extrême gau-

81. Johsua F., Du Cleuziou Y., « Raison. Entre radicalité et apolitisme », in Agrikoliansky E., Sommier I., Radiographie du mouvement altermondialiste, op. cit., p. 251.

82. Sommier I., Les Nouveaux mouvements contestataires, op. cit., p. 50.

83. Erdi Lelandais G., "Cosmopolites enracinés". La transnationalité et la construction identitaire du mouvement altermondialiste. L'Exemple de la Turquie, op. cit. 
che, soit en raison de leurs opinions ou de leurs productions intellectuelles. La plus emblématique de ces figures est sans doute Fikret Baskaya, qui a accumulé de nombreuses peines et qui continue à être condamné par la justice turque en raison de ses articles et ouvrages ${ }^{84}$. Porte-parole de la GDO'ya Hayir Platformu (Plateforme contre les OGM), Levent Alev, désormais ardent militant de l'agriculture biologique, a été membre du Dev-Yol et a fait de la prison après le coup d'Etat de 1980 85. S'agissant de militants actifs, nous observons donc un effet générationnel et une reconversion d'anciens militants dans le mouvement altermondialiste. Des « études longitudinales 86 » réalisées auprès d'anciens militants des années 1960 ont montré que :

"plutôt que de changer d'opinion politique en vieillissant, la plupart restaient attachés aux valeurs et tendances politiques qu'ils avaient développées dans leur jeunesse et leurs expériences générationnelles restaient l'un des thèmes dominants de leur vie à mesure qu'ils avançaient dans la vie ${ }^{87}$ ».

La composition des militants dans le mouvement turc confirme ce constat. La plupart de ces militants sont issus de 1968 et portent encore la nostalgie des mouvements de cette époque, qui avaient trouvé un soutien massif de la part de la population. Le mouvement altermondialiste représente pour eux une résurrection, une nouvelle possibilité de constituer un mouvement jeune, proche de celui des années 1970. En effet, ceux qui ont appartenu à cette génération politique ressentent encore pendant longtemps l'intensité de l'expérience et les attitudes et comportements politiques ultérieurs des anciens membres du mouvement présentent nombre de continuités avec leur passé politique. Leur désillusion envers la lutte politique joue également un rôle important dans leur participation à l'altermondialisme plus souple, moins visible, mais motivant et englobant. Elle est en même temps moins risquée en tant que lutte politique puisqu'elle ne nécessite pas forcément d'affrontement permanent avec l'ordre politique établi en Turquie. Ils se trouvent donc en retrait vis-àvis de leurs engagements passés, qu'ils s'en éloignent, qu'ils y reviennent ou qu'ils se maintiennent à distance d'eux. Ce retrait s'accompagne d'une mise à distance de la politique en général, suggérant que la cause altermondialiste leur offre un moyen de retrouver des activités politiques tout en maintenant une distance aux règles et enjeux de la politique 88 .

84 . Il a été condamné en 2001 à 16 mois de prison ferme suite à la parution de son livre intitulé $L a$ Faillite du paradigme, qui critiquait l'idéologie nationaliste-kémaliste dominante dans l'appareil étatique.

85 . Entretien réalisé avec L. Alev, 8 avril 2005, Istanbul.

86. Etudes réalisées sur 10-20 ans pour observer les attitudes d'une génération.

87. Crete J., Favre P. (dirs.), Générations et politiques, Paris, Economica, 1989, p. 25.

88. Fillieule O., Blanchard P., "Carrières militantes et engagement contre la globalisation », in Agrikoliansky E., Sommier I., op. cit., p. 177. 
Pour conclure, le champ militant en Turquie a toujours eu une relation symbiotique avec le champ politique. L'autonomisation difficile de ce champ a fait en sorte que tous les mouvements sociaux influents et les mobilisations d'envergure dans l'histoire des luttes sociales soient subordonnés à des courants politiques, voire à des partis politiques. Cet attachement n'est pas propre aux mouvements de gauche, on l'observe également dans les mouvements nationalistes, notamment les «Loups gris ». L'emprise du champ politique sur les mouvements sociaux perdure donc toujours.

Néanmoins, comme nous l'avons montré dans notre troisième partie, malgré la présence des partis politiques et la place indispensable des idéologies à la fois au sein de l'altermondialisme et à l'intérieur des organisations qui le composent, nous observons depuis une dizaine d'année l'émergence de certains mouvements comme le mouvement de Bergama, le mouvement antinucléaire ou la Plateforme anti-OGM dans lesquels l'idéologie n'a pas le rôle principal. L'altermondialisme contribue désormais à cette transformation grâce aux liens transnationaux qu'il développe et aux habitudes de mobilisation que les organisations turques observent chez leurs homologues étrangers, et notamment européens ${ }^{89}$. L'émergence de coordinations souples et peu hiérarchisées heurte l'héritage de normes traditionnelles, prônant le culte de l'organisation. Mais la transformation du militantisme grâce aux campagnes altermondialistes est également une réalité non négligable. Reste à savoir si, dans le futur, ce potentiel de l'altermondialisme va permettre de créer une mobilisation de masse et de renouveler les pratiques militantes, tout en assurant une relative autonomie du champ militant vis-à-vis du champ politique.

89. Erdi Lelandais G., "Cosmopolites enracinés". La transnationalité et la construction identitaire du mouvement altermondialiste. L'Exemple de la Turquie, op. cit., chapitre 3. 


\section{Annexes}

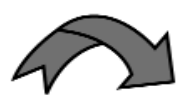

STRUCTURES CRÉÉES

APRÈS L'APPARITION

COORDINATIONS:

DE L'ALTERMONDIALISME :

- Plateforme Anti-OGM

- Küresel BAK

- Halkevleri

- Forum social d'Istanbul

- Mazlum-Der

- Özgür-Der

- Coordination anti-guerre

- Tribunal mondial de guerre pour l'Irak

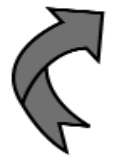

SYNDICATS ET CHAMBRES

PROFESSIONNELLES

- KESK

- DISK

- TMMOB

- TTB

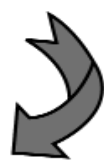

PARTIS POLITIQUES :

- DSIP

- ANTIKAPITALIST

- EMEP

- ÖDP

- DTP

\section{La Galaxie altermondialiste turque}

DSIP : Parti révolutionnaire socialiste des ouvriers - tendance trotskyste.

Antikapitalist : Scission du DSIP en 1997 - tendance trotskyste.

EMEP : Parti du travail - tendance marxiste, léniniste orthodoxe.

ÖDP : Parti de la liberté et de la solidarité - tendance socialiste avec plusieurs courants.

DTP : Parti de la Turquie démocratique - parti défendant les droits du peuple kurde.

TMMOB : Union des chambres des ingénieurs et des architectes de Turquie proche des partis de gauche.

TTB : Union des médecins de Turquie - proche des partis de gauche.

Halkevleri : Transformation des mouvements politiques communistes des années 1970 en association, marxiste orthodoxe. 
Coordination anti-guerre : Fondée pour prévenir la guerre en Irak, elle a organisé de nombreuses manifestations pour empêcher l'envoi de troupes turques en Irak.

Küresel BAK: Coalition de paix de justice globale, scission de la Coordination, proche du DSIP et de l'ÖDP.

Forum social d'Istanbul : fondé en 2002 à l'initiative des membres de l'ÖDP et du DSIP pour coordonner les activités liées aux processus des forums sociaux. Ils ont organisé en octobre 2006 le Forum social de Turquie.

Tribunal mondial de guerre pour l'Irak : ce projet a été lancé en 2003 par la fondation Bertrand Russell pour la paix à Bruxelles et le groupe turc s'est vu attribuée la responsabilité du secrétariat et la coordination entre les groupes de Bruxelles, d'Hiroshima, de New-York et de Londres. Ce projet international, réalisé à Istanbul en juin 2005, vise à clarifier les crimes de guerre commis en Irak par les soldats Américains et à organiser un procès entièrement symbolique. 